\title{
ACOUSTIC ANALYSIS WITH VOCAL LOADING TEST IN OCCUPATIONAL VOICE DISORDERS: OUTCOMES BEFORE AND AFTER VOICE THERAPY
}

\author{
${ }^{1}$ Nofer Institute of Occupational Medicine, Łódź, Poland \\ Department of Audiology and Phoniatrics \\ ${ }^{2}$ Nofer Institute of Occupational Medicine, tódź, Poland \\ Department of Physical Hazards
}

EWA NIEBUDEK-BOGUSZ ${ }^{1}$, PIOTR KOTYŁO ${ }^{1}$, PIOTR POLITAŃSKI ${ }^{2}$, and MARIOLA ŚLIWIŃSKA-KOWALSKA ${ }^{1,2}$

\begin{abstract}
Objective: To assess the usefulness of acoustic analysis with vocal loading test for evaluating the treatment outcomes in occupational voice disorders. Methods: Fifty-one female teachers with dysphonia were examined (Voice Handicap Index - VHI, laryngovideostroboscopy and acoustic analysis with vocal loading) before and after treatment. The outcomes of teachers receiving vocal training (group I) were referred to outcomes of group II receiving only voice hygiene instructions. Results: The results of subjective assessment (VHI score) and objective evaluation (acoustic analysis) improved more significantly in group I than in group II. The post-treatment examination revealed a decreased percentage of subjects with deteriorated jitter parameters after vocal loading, particularly in group I. Conclusions: Acoustic analysis with vocal loading test can be a helpful tool in the diagnosis and evaluation of treatment efficacy in occupational dysphonia.
\end{abstract}

Key words:

Occupational voice disorders, Vocal loading, Objective assessment, Voice therapy

\section{INTRODUCTION}

Occupational voice disorders affect the quality of life and the career of professional voice users as well as bring about decreased profits to their employers [1]. Excessive use or abuse of one's voice at work can lead to a number of symptoms, including hoarseness, soreness, weak voice, vocal fatigue or even aphonia [2]. It has been suggested that teachers are more at risk of developing voice disorders than others $[3,4]$. Roy et al. reported the prevalence of lifetime voice disorders to be significantly higher among teachers (57.7\%) than in non-teachers $(28.8 \%)$ [5].

The work-related organic larynx pathologies are frequently preceded by long-lasting non-organic voice disorders (functional dysphonia). Non-treated functional dysphonia often leads to irreversible larynx lesions, such as polypoid hypertrophia or vocal nodules, which disturb voice condition [6]. The Polish study demonstrated that the main risk factors for developing occupational dysphonia were lifetime vocal effort, incorrect techniques of voicing, and psychological predisposition [7]. The prevention and treatment of occupational voice disorders calls for improved occupational safety and health arrangements for voice and speech professionals [8]. Moreover, the knowledge about voice care and hygiene among dysphonic subjects is still inadequate [9].

A precise evaluation of the vocal function in professional voice users is a major problem in diagnosing dysphonia [10,11]. The clinical methods include

Parts of the study results were presented at the 7th Pan European Voice Conference, Groningen, The Netherlands, August 29-September 12007 . This study was supported by the project of the Polish State Committee for Scientific Research, Project No 18.6/06.

Received: March 27, 2008. Accepted: October 29, 2008.

Address reprint requests to Ewa Niebudek-Bogusz, Department of Audiology and Phoniatrics, Nofer Institute of Occupational Medicine, ul. św. Teresy 8, 91-348 Łódź, Poland (e-mail: ebogusz@imp.lodz.pl). 
laryngovideostroboscopy, aerodynamic test, glottography, acoustic analysis, as well as self-assessment of voice disorder (e.g. Voice Handicap Index) [12-14]. Among these instruments, objective voice evaluation via acoustic measurement seems to be of particular value because it is noninvasive and relatively easy to perform $[15,16]$. It is also important to evaluate the efficacy of treatment in teachers' voice disorders. Some authors demonstrate the usefulness of acoustic tests for evaluating the progress in voice therapy $[17,18]$. In the teaching professions, the examination of the voice endurance may play a role in estimating voicerelated working ability. Therefore, the evaluation of vocal loading with an objective test such as acoustic analysis is particularly meaningful.

The aim of this study was to assess the usefulness of acoustic analysis with vocal loading test for evaluating the outcomes of phoniatric treatment of functional voice disorders.

\section{MATERIALS AND METHODS}

The study concerned 51 professionally active full-time female teachers (aged 28 to 57 years, mean 41) with functional voice disorders (non-organic dysphonia) treated at Voice Clinic (outpatient), Nofer Institute of Occupational Medicine, Łódź, Poland. The patients received over a three months' long phoniatric treatment consisting in vocal hygiene instructions, vocal training, moisturizing inhalations, humidification and/or vitamin supplementation. Six of the patients who failed to complete the procedure and attend the control examination were excluded from the study. The subjects were 46 teachers classified into two groups: 30 subjects received both vocal training and voice hygiene instructions (group I) and the remaining 16 received only voice hygiene instructions (group II). They were teachers of elementary and secondary schools, most of them were language teachers (32\% subjects) and primary education teachers (28\% subjects). The number of teaching hours per week ranged from 18 to $30 \mathrm{~h}$ (mean $21.4 \mathrm{~h}$ ). The characteristics of the study groups is summarized in Table 1. The groups did not differ significantly with respect to age, years of teaching experience, and number of teaching
Table 1. Characteristics of the study groups

\begin{tabular}{cccc}
\hline & $\begin{array}{c}\text { Age } \\
\text { (years) }\end{array}$ & $\begin{array}{c}\text { Employment time } \\
\text { (years) }\end{array}$ & $\begin{array}{c}\text { Number of school } \\
\text { hours per week }\end{array}$ \\
\hline $\begin{array}{c}\text { Group I } \\
\mathrm{n}>30\end{array}$ & 40.0 & 15.9 & 21.9 \\
$\begin{array}{r}\text { Group II } \\
\mathrm{n}>16\end{array}$ & 41.4 & 16.6 & 20.8 \\
\hline
\end{tabular}

Group I received vocal training and voice hygiene instructions. Group II received voice hygiene instructions only.

Table 2. Diagnostic categories detected in study groups

\begin{tabular}{lccc}
\hline \multicolumn{1}{c}{ Diagnosis } & $\begin{array}{c}\text { Group I } \\
\mathrm{n}=30\end{array}$ & $\begin{array}{c}\text { Group II } \\
\mathrm{n}=16\end{array}$ & $\begin{array}{c}\text { Total } \\
\mathrm{n}=46\end{array}$ \\
\hline $\begin{array}{c}\text { Soft vocal nodules } \\
\text { (prenodules) }\end{array}$ & 12 & 4 & 16 \\
$\begin{array}{c}\text { Glottal insufficiency } \\
\text { (MTP I) }\end{array}$ & 10 & 5 & 15 \\
$\begin{array}{c}\text { Hyperfunctional } \\
\text { dysphonia (MTP II) }\end{array}$ & 5 & 4 & 9 \\
\begin{tabular}{l} 
Others \\
\hline
\end{tabular} & 3 & 3 & 6 \\
\hline
\end{tabular}

MTP — Muscle Tension Pattern.

hours per day. The groups did not differ also in severity and diagnostic categories of voice disorders (Table 2). For ethical reasons, no control group without any treatment was established.

All the participants were examined twice: before the treatment (initial examination) and after the period of treatment (control examination). The examination procedure covered the Voice Handicap Index (VHI) questionnaire (Polish version according to Pruszewicz [19]), and laryngologic-phoniatric examination, followed by laryngovideostroboscopy (LVSS) and voice acoustic analyses. Acoustic analysis were performed before and after vocal loading test (reading aloud a text for 30 min under exposure to $85 \mathrm{dBSPL}$ white noise) both in the initial and control examination (Fig. 1). The procedure of laryngovideostroboscopy, acoustic analysis and vocal loading test were described in detail elsewhere [20]. The following parameters were estimated:

1. Frequency perturbation parameters: Jitter (Pitch perturbation), RAP (Relative Average Perturbation) and PPQ (Pitch Perturbation Quotient). 
2. Amplitude perturbation parameters: shimmer (Amplitude perturbation) and APQ (Amplitude Perturbation Quotient).

3. Noise evaluation measurement: NHR (Noise to Harmonic Ratio).

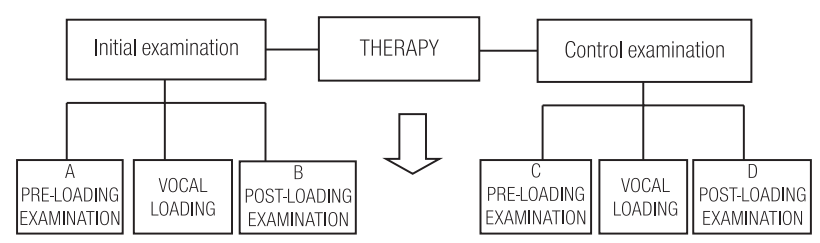

Fig. 1. Study protocol scheme.

When comparing the results before and after vocal loading, the change observed in acoustic analysis was classified using a binary system either as $1-$ deterioration or $0-$ no deterioration. An outcome was regarded as a deterioration in respective acoustic parameter if its value at the post-loading examination increased more than by a standard deviation in comparison to its value recorded at the pre-loading examination. At the post-therapy examination, the videostroboscopic imaging was regarded as an improvement if at least one parameter was evaluated by both ENT phoniatricians as better than in the pre-therapy examination. The pre- and post-therapy voice assessment involved also determining the maximum phonation time (MPT), a simple but very useful quantitative aerodynamic parameter.

Vocal training (performed in group I only) was conducted by two experienced speech-language pathologists according to a standardized vocal training protocol once a week, each session lasting for $45 \mathrm{~min}$. The vocal training programme comprised four main elements:

a) breathing and relaxation exercises,

b) vocal function exercises,

c) resonant improvement exercises,

d) carryover exercises,

and lasted for an average period of 3 months.

The subjects from both groups received instructions concerning vocal hygiene, which covered advice how to eliminate voice abuse/misuse, avoid irritants an maintain adequate hydration of mucosa of the voice organ. Other details of direct and indirect voice therapy have been described elsewhere [21].

\section{Ethics}

This study was approved by the Ethics Committee at the Nofer Institute of Occupational Medicine in Łódź.

\section{Statistical analysis}

The following statistical methods were used to analyse the data:

1. Chi-square significance test for within- and betweengroup comparisons of the frequency or distribution of discrete variables (such as the number of abnormal results, number of changes in parameter values, values above and below normal, and vocal-loading effect on voice stability, measured as the changes in the value of each parameter before and after the vocal loading test.

2. The Wilcoxon signed-rank test to compare the stability of acoustic parameters (changes related to vocal loading test) estimated at the pre- and post-treatment examination, and also to compare the percentage of subjects with pre- and post-treatment changes in videostroboscopic parameters.

3. T-Student test to compare the mean values of VHI (total score and subscale score) between two groups.

4. Multi-way analysis of variance (MANOVA) to compare the effects of therapy on VHI results, MPT and acoustic parameters; it was used to estimate the significance of changes in acoustic parameters in relation to vocal loading test at two time-points in two study groups. The SPSS software was used for all the analyses. $\mathrm{P}<0.05$ was considered statistically significant.

\section{RESULTS}

In the study population, the phoniatric examination with LVSS revealed that the most frequent larynx pathology were reversible soft vocal nodules (prenodules) found in $34.8 \%$ of subjects (Table 2). These were followed by glottal insufficiency (MTP I - Muscle Tension Pattern type I with a longitudinal gap between the vocal free edges during phonation, acc. to Koufman et al. [22]) in 32.6\% of subjects. Ranked third was hyperfunctional dysphonia with a tendency for vestibular voice (MTP II - Muscle 
Tension Pattern type II with approximation of the vestibular vocal folds acc. to Koufman et al. [22]) in $19.6 \%$ of subjects. The distribution of diagnosed pathologies in both groups is presented in Table 2.

\section{Phoniatric examination with LVSS \\ before and after therapy (A vs. C)}

The assessment of maximum phonation time (MPT) revealed that in group I who attended vocal training, this quantitative parameter increased significantly $(p>0.046)$ : from $14.3 \mathrm{~s}$ at the initial examination to $17.4 \mathrm{~s}$ at the control examination. Such improvement could not be observed in group II receiving only voice hygiene instructions. The comparison of qualitative laryngovideostroboscopic (LVSS) parameters at the initial and control examination showed that in group I, a significant post-therapy improvement was noted for the following parameters: regularity of vocal fold vibration ( $p>0.008)$, amplitude of vocal fold movement $(p>0.032)$ and quality of mucosal wave ( $p>0.002)$. However, in group II, an improvement of two of the videostroboscopic parameters: amplitude $(p>0.039)$ and mucosal wave ( $p>0.023$ ) was also observed. The LVSS imaging was evaluated by two ENT phoniatricians, but the inter- and intra-judge reliability had not been investigated. Consequently these results have not been considered in the discussion. LVSS was used not only to evaluate the vocal fold status before and after the therapy, but also to adapt the vocal training protocol to individual needs.

\section{Acoustic analysis before and after therapy (A vs. C)}

At the pre-treatment examination, the results of acoustic analysis in the two groups did not differ significantly. The acoustic parameters showed better values at the posttreatment examination, particularly in group I. However, the mean fundamental frequency of speaking voice (F0) in both the groups was lower than the respective value for euphonic women of similar age $(235 \mathrm{~Hz}$ - own data [23]). F0 significantly increased in group I only $(211 \mathrm{~Hz}$ vs. $221 \mathrm{~Hz}$ ) and approximated the normal value. At the control examination, some other acoustic parameters were found to improve more significantly, especially in group I. These were jitter: $p>0.000$, RAP: $p>0.001$ and PPQ:
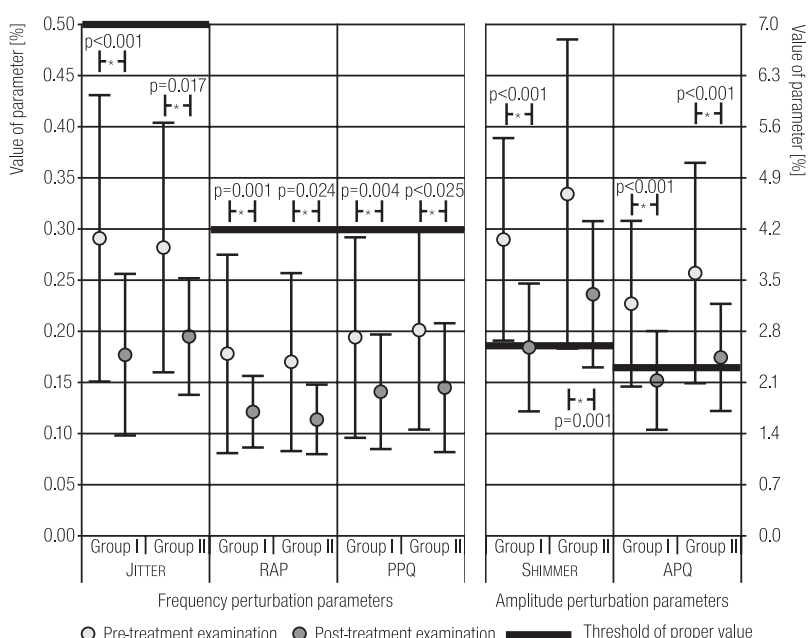

Fig. 2. Frequency perturbation parameters: Jitter, RAP and PPQ and amplitude perturbation parameters: Shimmer and $\mathrm{APQ}$ at pre- and post-treatment examination in both groups.

Table 3. Comparison of acoustic parameters at the initial and control examination in both study groups

\begin{tabular}{|c|c|c|c|c|c|c|c|}
\hline \multirow{4}{*}{ Parameter } & \multirow{4}{*}{ Threshold of normal value } & \multicolumn{3}{|c|}{ Group I } & \multicolumn{3}{|c|}{ Group II } \\
\hline & & Initial & Control & \multirow{3}{*}{$\mathrm{P}$} & Initial & Control & \multirow{3}{*}{$\mathrm{P}$} \\
\hline & & \multicolumn{2}{|c|}{ Examination } & & \multicolumn{2}{|c|}{ Examination } & \\
\hline & & A & $\mathrm{C}$ & & A & $\mathrm{C}$ & \\
\hline Jitter & 0.5 & 0.29 & 0.177 & $0.000^{*}$ & 0.281 & 0.194 & $0.017^{*}$ \\
\hline RAP & 0.3 & 0.177 & 0.113 & $0.001^{*}$ & 0.169 & 0.113 & $0.024 *$ \\
\hline PPQ & 0.3 & 0.193 & 0.140 & $0.004^{*}$ & 0.200 & 0.144 & $0.025^{*}$ \\
\hline Shimmer & 2.6 & 4.057 & 2.580 & $0.000^{*}$ & 4.681 & 3.306 & $0.001^{*}$ \\
\hline APQ & 2.3 & 3.177 & 2.127 & $0.000^{*}$ & 3.600 & 2.444 & $0.000^{*}$ \\
\hline NHR & 3.4 & 3.427 & 2.093 & $0.000^{*}$ & 3.563 & 2.063 & $0.003^{*}$ \\
\hline
\end{tabular}

$* p<0.05$ 


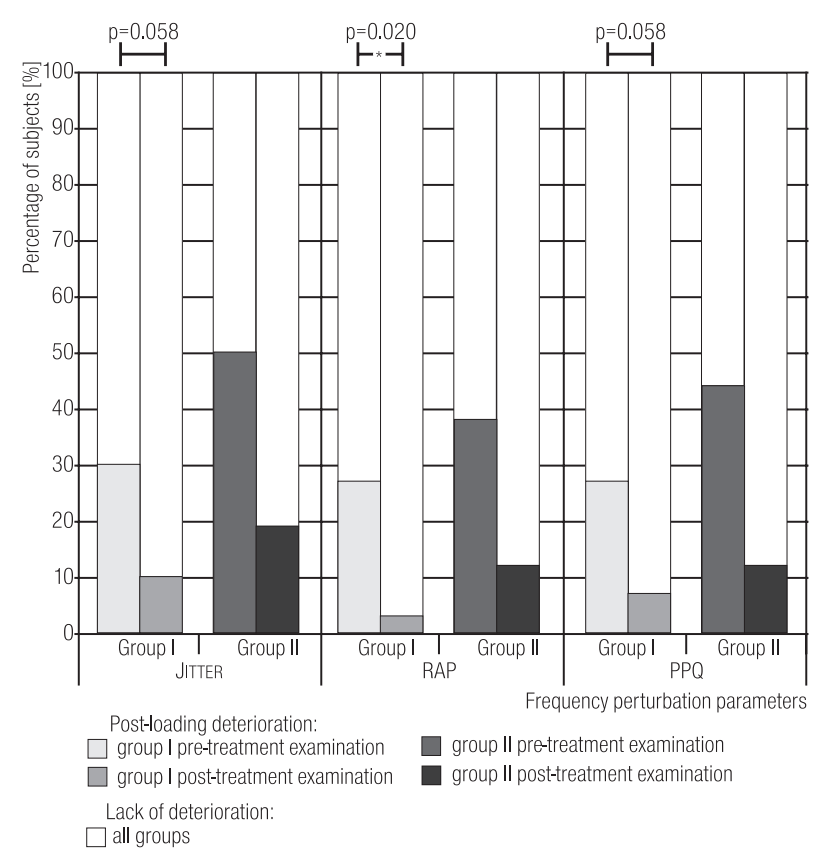

Fig. 3. Vocal loading effect on frequency perturbation parameters (jitter group) at pre- and post-treatment examination in both groups.

p > 0.004 (Table 3). However, one should note that they did not exceed their normal threshold values at the initial examination (Fig. 2). The mean amplitude perturbation measures (shimmer, APQ) initially showed pathological values in both groups (Table 3), while at the control examination these parameters improved and were around their normal limits in group I (Fig. 2).

\section{Vocal loading effect on acoustic parameters before and after therapy (B-A vs. D-C)}

Before the treatment, vocal loading resulted in a slight deterioration in the frequency perturbation parameters (jitter, RAP, PPQ) in both groups. The post-treatment examination revealed the tendency of post-loading improvement of all these parameters in group I, and post-loading deterioration of jitter and RAP in group II. However, these changes were not statistically significant. The finding above did not refer to amplitude perturbation parameters (shimmer and APQ) and NHR. Moreover, the percentage of subjects in group I in whom the frequency perturbation parameters deteriorated after the vocal loading in the post-treatment examination decreased, compared to the initial examination (Fig. 3). For RAP, this difference was statistically significant $(\mathrm{p}>0.02)$ while for jitter and $P P Q$, it was of borderline significance ( $p>0.058$ ). No significant changes were observed in group II. These differences in the post-loading effect did not refer to amplitude perturbation parameters (shimmer and APQ) in any of the groups.

\section{Subjective assessment by Voice Handicap Index before and after therapy (A vs. C)}

The pre- and post-treatment results of acoustic analysis with vocal loading test in teachers were referred to the outcomes to the subjective assessment. At the pre-treatment examination, the results of $\mathrm{VHI}$ and acoustic analysis in the two groups did not differ significantly. At the posttreatment examination, group I attending vocal training showed significantly better VHI results compared to the initial findings. This referred to the total score $(p<0.000)$ and to each of the VHI subscales: functional ( $p>0.003)$, emotional ( $p>0.003)$, and physical ( $p>0.001)$. In group II, which received only vocal hygiene instructions, the total VHI score improved to a lesser degree and only on the physical subscale.

\section{DISCUSSION}

The ability to assess voice disorders has improved dramatically over the last two or three decades. Despite the introduction of many new diagnostic methods and techniques, there is no commonly adopted 'gold standard' for voice examination. Multidimensional assessment of voice pathology is recommended; this includes perception, laryngovideostroboscopy, acoustics, aerodynamics, and subjective rating by the patient [24,25]. Many voice disorders, particularly the work-related ones, are associated with vocal loading, but a direct measurement of the effect of vocal fatigue has not been possible thus far. Moreover, evaluating vocal loading effect in professional voice users is of clinical importance [8]. The aim of this study was to assess the applicability of acoustic analysis with the vocal loading test to the evaluation of treatment outcomes in occupational dysphonia. 
The acoustic analysis of voice is commonly used in voice quality evaluation and treatment assessment [26,27]. The present study showed a post-therapy improvement in acoustic parameters, particularly in group I. The increase in the mean F0 value noted in group I at the control examination should be interpreted as a sign of voice quality improvement in view of the recent reports that in female teachers with voice disorders, the average F0 value is lower than the normal threshold [28,29]. The analysis of the other acoustic parameters also showed a post-treatment improvement in both study groups. A particularly positive effect of the therapy was noted in group I (for all six parameters: jitter, RAP, PPQ, shimmer, APQ and NHR, $\mathrm{p}<0.001)$. Moreover, the pre-therapy shimmer and APQ exceeded their normal threshold values in both groups, in the post-therapy examination these parameters were within their normal limits in group I.

Similar results were reported by Speyer et al. [30]. They compared pre- and post-therapy data for three acoustic parameters: jitter, shimmer and NHR, and also found a posttherapy improvement for these parameters ( $\mathrm{p}<0.003)$. They stressed that in order to evaluate the overall effect of voice therapy, a multidimensional voice examination should be considered. The results of pre- and post-therapy acoustic analysis were referred to other methods of voice assessment: self-rating scale (VHI), aerodynamic measurement (MPT), and laryngovideostroboscopy. The treatment of dysphonia in group I was found to be more effective with respect to the emotional and functional subscales of VHI. The aerodynamic parameter of MPT improved significantly only in group I; MPT increase in group II was non-significant.

Additionally, vocal loading effect was examined with acoustic analysis. The post-therapy examination revealed a decreased percentage of subjects in whom the frequency perturbation parameters (jitter group) deteriorated after vocal loading, compared to the pre-therapy results; this refers particularly to group I. This change was statistically significant for RAP $(p>0.02)$ while for jitter and PPQ it was of borderline significance ( $p>0.058)$ only in group I. These acoustic parameters represented a relative period-to-period variability of the frequency. This data may point to a post-training decrease in pitch variability in group I and indicate that vocal training may play a role in protecting the laryngeal organ against undesirable changes related to vocal loading. This could be associated with a vocal warm-up due to vocal effort and an acquired ability to compensate for vocal fatigue. These results indicate that voice stability improvement may be the effect of vocal training, which is of special significance in the light of the reports on voice instability as a characteristic trait of functional dysphonia [31]. However, Laukanen et al. [32] stressed that voice evaluation should accommodate the possible individual susceptibility to vocal fatigue.

\section{CONCLUSIONS}

The quantitative method of voice acoustic analysis performed before and after vocal loading test seems to be an effective complement to objective voice examinations in professional voice users as well as a helpful tool for evaluating the treatment of occupational dysphonia. It should be stressed that voice disorders affect a very large professional group. Therefore, their prevention and early detection is of particular clinical importance.

\section{REFERENCES}

1. Titze IR, Lemke J, Montequin D. Populations in the U.S. workforce who rely on voice as a primary tool of trade: a preliminary report. J Voice 1997;11(3),254-9.

2. Verdolini K, Ramig LO. Review: occupational risks for voice problems. Logoped Phoniatr Vocol 2001;26:37-46.

3. Roy N, Merrill RM, Thibeault S, Gray SD, Smith EM. Voice disorders in teachers and the general population: effect on work performance, attendance, and future career choices. J Speech Lang Hear Res 2004;47:542-51.

4. Smith EM, Gray SD, Dove H, Kirchner HL, Heras H. Frequency and effects of teachers' voice problems. J Voice 1997;11(1):81-7.

5. Roy N, Merrill RM, Thibeault S, Parsa RA, Gray SD, Smith EM. Prevalence of voice disorders in teachers and the general population. J Speech Lang Hear Res 2004;47(2): 281-93. 
6. Rubin JS, Sataloff RT, Korovin GS, editors. Diagnosis and treatment of voice disorders. New York: Thomson Delmar Learning; 2003.

7. Sliwinska-Kowalska M, Niebudek-Bogusz E, Fiszer M, LosSpychalska T, Kotylo P, Sznurowska-Przygocka B, et al. The prevalence and risk factors for occupational voice disorders in teachers. Folia Phoniatr Logop 2006,58(2):85-101.

8. Vilkman E. Occupational safety and health aspects of voice and speech professions. Folia Phoniatr Logop 2004,56(4):220-53.

9. Dejonckere PH. Occupational voice-care and cure, Hague: Kugler Publications; 2001.

10. Niebudek-Bogusz E, Fiszer M, Kotyło P, Sliwinska-Kowalska M. Diagnostic value of voice analysis in assessment of occupational voice pathologies in teachers. Logoped Phoniatr Vocol 2006;31:100-6.

11. Hoffman-Ruddy B, Lehman J, Crandell C, Ingram D, Sapienza C. Laryngostroboscopic, acoustic, and environmental characteristics of high-risk vocal performers. J Voice 2001;15(4);543-52.

12. Awan SN, Roy N. Acoustic prediction of voice type in women with functional dysphonia. J Voice 2005;19(2):268-82.

13. Obrebowski A. The voice organ and its role in social communication. 1st ed. Poznań: Akademia Medyczna; 2008 [in Polish].

14. Rosen CA, Murry T, Zinn A, Zullo T, Sonbolian M. Voice handicap index change following treatment of voice disorders. J Voice 2000;14:619-23.

15. Jónsdottir V, Laukkanen AM, Vilkman E. Changes in teachers' speech during a working day, with and without electric sound amplification. Folia Phoniatr Logop 2002;54(6): $282-7$.

16. Yu P, Ouaknine M, Revis J, Giovanni A. Objective voice analysis for dysphonic patients: A multiparametric protocol including acoustic and aerodynamic measurements. J Voice 2001;15(4):529-42.

17. McCrea CR, Morris RJ. Effects of vocal training and phonatory task on voice onset time. J Voice 2007;21(1):54-63.

18. Roy N, Hendarto H. Revisiting the pitch controversy: changes in speaking fundamental frequency (SFF) after management of functional dysphonia. J Voice 2005;19(4):582-91.

19. Pruszewicz A, Obrebowski A, Wiskirska-Woznica B, Wojnowski W. The complex voice assessment — Polish version of the Voice Handicap Index (VHI). Otolaryngol Pol 2004; 58(3):547-9 [in Polish].

20. Niebudek-Bogusz E, Kotylo P, Sliwińska-Kowalska M. Evaluation of voice acoustic parameters related to vocal-loading test in professionally active teachers with dysphonia. Int J Occup Med Environ Health 2007;20(1):25-30.

21. Niebudek-Bogusz E, Sznurowska-Przygocka B, Fiszer M, Kotylo P, Sinkiewicz A, Modrzewska M, et.al. The effectiveness of voice therapy for teachers with dysphonia. Folia Phoniatr Logop 2008;60(3):107-62.

22. Koufman JA. Laryngeal muscle tension patterns (MTPs). In: Rubin JS, Sataloff RT, Korovin GS, editors. Diagnosis and treatment of voice disorders. New York: Thomson Delmar Learning; 2003. p. 175-82.

23. Niebudek-Bogusz E, Fiszer M, Kotyło P, Just M, ŚliwińskaKowalska M. Voice acoustic analysis in healthy women. Otolarynolaryngol Przegl Klin 2004;3(1),1-12. [in Polish]

24. DeJonckere PH, Crevier-Buchman L, Marie JP, Moerman M, Remacle M, Woisard V. Implementation of the European Laryngological Society (ELS) basic protocol for assessing voice treatment effect. Rev Laryngol Otol Rhinol 2003;124:279-83.

25. Simberg S, Sala E, Tuomainen, Sellman J, Rönnemaa AM. The effectiveness of group therapy for students with mild voice disorders: a controlled clinical trial. J Voice 2006;20(1): 97-109.

26. Gillivan-Murphy P, Drinnan MJ, O’Dwyer TP, Ridha H, Carding P. The effectiveness of voice treatment approach for teachers with self-reported voice problems. J Voice 2006;20(3):423-31.

27. Timmermans B, de Bodt MS, Wuyts FL, van de Heyning PH. Training outcome in future professional voice users after 18 months of voice training. Folia Phoniatr Logop 2004;56(2):120-9.

28. Domeracka-Kołodziej A, Maniecka-Aleksandrowicz B, Grzanka A. Voice quality in teachers with occupational voice disorders. Otolarynolaryngol Przegl Klin 2002;1(2):105-12 [in Polish].

29. Tavares EL, Martins RH. Vocal evaluation in teachers with or without symptoms. J Voice 2007;21(4):407-14.

30. Speyer R, Wieneke GH, Dejonckere PH. Documentation of progress in voice therapy: perceptual, acoustic, and 
laryngostroboscopic findings pretherapy and posttherapy. J Voice 2004;18(3):325-40.

31. Jilek C, Marienhagen J, Hacki T: Vocal stability in functional dysphonic versus healthy voices at different times of voice loading. J Voice 2004;18(4):443-53.
32. Laukkanen AM, Järvinen K, Artkowski M, Waaramaa-Mäki-Kulmala T, Kankare E, Sippola S, et al. Changes in voice and subjective sensations during a 45-min vocal loading test in female subjects with vocal training. Folia Phoniatr Logop 2004;56(6):335-46. 